\title{
New IBD genes?
}

\section{McGovern, T Ahmad}

\section{New reports of potential novel inflammatory bowel disease (IBD) genes further advance our understanding of the genetic basis of IBD}

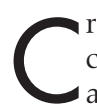
rohn's disease $(C D)$ and ulcerative colitis result from an inappropriate response of the mucosal immune system to the normal enteric flora in a genetically susceptible individual. The importance of genetic predisposition was firmly established in 2001 by the identification of the first $C D$ susceptibility gene NOD2 (CARD15) on chromosome $16 q 12$ (IBDl). ${ }^{12}$ It is now clear that NOD2 variants contribute only a small part to genetic susceptibility, suggesting the existence of other, as yet unidentified, genes. Although these have remained elusive, linkage studies have provided important clues to their location, implicating at least seven other regions of the human genome.

Two recent studies report the cloning of potential novel inflammatory bowel disease (IBD) genes within linkage areas on chromosomes $5^{3}$ and $10 .^{4}$ Linkage between $\mathrm{CD}$ and chromosome 5q31-33 (IBD5) was first demonstrated in $1999^{5}$ and further characterisation of this locus refined the region to a highly conserved $250 \mathrm{~kb}$ haplotype. ${ }^{6}$ Identifying the IBD5 causal mutation(s) has been hampered by the degree of linkage disequilibrium and the density of immunoregulatory genes on this haplotype. A Canadian group $^{3}$ now suggest that the true IBD5 disease causing mutations occur in SLC22A4 and SLC22A5, encoding OCTN1 and OCTN2 respectively, members of the organic cationic transporter subfamily. These proteins are involved in the elimination of toxins and the uptake of various physiological substrates, including carnitine (required for oxygen burst mediated pathogen killing). OCTNI and OCTN2 are widely expressed, including in intestinal epithelial cells, macrophages, and $\mathrm{T}$ cells. Two putatively functional single nucleotide polymorphisms (SNPs), one in SLC22A4 and the other in SLC22A5, form a risk haplotype enriched in individuals with $C D$. The L503F (leucine to phenylalanine at codon 503 ) polymorphism maps to a region of SLC22A4, important for cellular transport, and functional data presented by the authors suggest the variant allele may affect uptake of carnitine, various xenobiotics, and toxins. The SLC22A5 SNP is located in the gene promoter where it appears to disrupt a predicted heat shock element required for the binding of heat shock transcription factors. Possession of

Peltekova VD, Wintle RF, Rubin LA, et al. Functional variants of OCTN cation transporter genes are associated with Crohn disease. Nat Genet 2004;36:471-5.

Crohn disease is a chronic, inflammatory disease of the gastrointestinal tract. A locus of approximately $250 \mathrm{~kb}$ at $5 \mathrm{q} 31$ (IBD5) was previously associated with susceptibility to Crohn disease, as indicated by increased prevalence of a risk haplotype of 11 single-nucleotide polymorphisms among individuals with Crohn disease, but the pathogenic lesion in the region has not yet been identified. We report here that two variants in the organic cation transporter cluster at $5 q^{31}$ (a missense substitution in SLC22A4 and a $G \rightarrow C$ transversion in the SLC22A5 promoter) form a haplotype associated with susceptibility to Crohn disease. These variants alter transcription and transporter functions of the organic cation transporters and interact with variants in another gene associated with Crohn disease, CARD15, to increase risk of Crohn disease. These results suggest that SLC22A4, SLC22A5 and CARD15 act in a common pathogenic pathway to cause Crohn disease.

Stoll M, Corneliussen B, Costello CM, ef al. Genetic variation in DLG5 is associated with inflammatory bowel disease. Nat Genet 2004;36:476-80.

Crohn disease and ulcerative colitis are two subphenotypes of inflammatory bowel disease (IBD), a complex disorder resulting from gene-environment interaction. We refined our previously defined linkage region for IBD on chromosome 10q23 and used positional cloning to identify genetic variants in DLG5 associated with IBD. DLG5 encodes a scaffolding protein involved in the maintenance of epithelial integrity. We identified two distinct haplotypes with a replicable distortion in transmission $(P=0.000023$ and $P=0.004$ for association with $I B D, P=0.00012$ and $\mathrm{P}=0.04$ for association with Crohn disease). One of the risk-associated DLG5 haplotypes is distinguished from the common haplotype by a nonsynonymous singlenucleotide polymorphism 113G $\rightarrow A$, resulting in the amino acid substitution $R 30 Q$ in the DUF622 domain of DLG5. This mutation probably impedes scaffolding of DLG5. We stratified the study sample according to the presence of risk-associated CARD15 variants to study potential gene-gene interaction. We found a significant difference in association of the 113A DLG5 variant with Crohn disease in affected individuals carrying the risk-associated CARD15 alleles versus those carrying non-riskassociated CARD15 alleles. This is suggestive of a complex pattern of gene-gene interaction between DLG5 and CARD15, reflecting the complex nature of polygenic diseases. Further functional studies will evaluate the biological significance of DLG5 variants.

the risk haplotype is associated with a 3-4-fold risk of disease, similar to that for possession of CARD15 mutations. Interestingly, the risk was much greater in the presence of both the risk haplotype and CD associated CARD15 alleles, consistent with an interaction between IBD5 and CARD15. The strong linkage disequilibrium across IBD5 makes interpretation of association data from individual SNPs difficult. This region is rich in genes, many of which are attractive candidates for disease pathogenesis. It therefore remains possible that other "functional" SNPs, expressed in tissues relevant to IBD, exist on this haplotype.
Precisely how these SNPs might interact with NOD2 to increase the risk of CD remains unclear. However, tantalising clues are provided by the suggestion that $\alpha$-defensins are potential substrates of OCTN1 and 2, and by a recent report suggesting that NOD2 variants may actually increase susceptibility to CD, through a defect in $\alpha$-defensin production. ${ }^{7}$ The same group has published a study in an independent cohort confirming their findings. Analyses in this cohort have revealed particular association with ileal $\mathrm{CD}^{8}$ in contrast to a previous study suggesting an association between IBD5 and perianal CD. 
A second study reports an association between SNPs in DLG5, a member of the membrane associated guanylate kinase gene family, and IBD. A German group ${ }^{4}$ performed linkage disequilibrium mapping of a locus on chromosome 10 and demonstrated association with variants in DLG5. Four common haplotypes across $D L G 5$ were identified. Haplotype $\mathrm{D}$ (containing the unique SNP 113A) conferred a modest risk of $C D$ while haplotype A was protective. The results were replicated in a case control study (but not in an independent family based association study) and once again possible interaction with the CD associated CARD15 alleles was noted. DLG5 is expressed in the colon and small intestine where it acts as a multifunctional adapter and scaffold protein involved in the regulation of epithelial cell growth, shape, and polarity. Computer prediction programs suggest that the IBD associated $D L G$ variants may impair this scaffolding function, but at present supportive data from functional experiments are not available. One published study has confirmed the association between CD and SNP113A (but not with the common haplotype) ${ }^{10}$ although two other studies have not been able to confirm CD association with DLG5. ${ }^{11}{ }^{12}$ Further large adequately powered studies in different populations are now required to assess the probable moderate effect of this gene in CD susceptibility.
The rapid advances in our understanding of the genetic basis of IBD are yet to impact on routine clinical practice although recent discoveries have provided considerable insights for investigators. There is considerable optimism that as IBD genes are identified and their environmental interactions unravelled that we may be able to better define the heterogeneous group of patients with IBD, aid understanding of the molecular mechanisms specific to subgroups of disease, and provide a framework to predict clinical phenotype and response to therapy. These novel findings reported by the Canadian and German make an important contribution towards this goal.

Gut 2005;54:1060-1061.

doi: 10.1136/gut.2004.048918

\section{Authors' affiliations}

D McGovern, T Ahmad, The Wellcome Trust

Centre for Human Genetics and

Gastroenterology Unit, University of Oxford,

Oxford, UK

Conflict of interest: None declared.

Correspondence to: Dr D McGovern, The Wellcome Trust Centre for Human Genetics, Roosevelt Drive, Headington, Oxford OX3 7BN, UK; dermot@well.ox.ac.uk

\section{REFERENCES}

1 Hugot JP, Chamaillard M, Zouali $\mathrm{H}$, et al Association of NOD2 leucine-rich repeat variants with susceptibility to Crohn's disease. Nature 2001;411:599-603.

2 Ogura $\mathrm{Y}$, Bonen DK, Inohara N, et al. A frameshift mutation in NOD2 associated with susceptibility to Crohn's disease. Nature 2001;411:603-6.

3 Peltekova VD, Wintle RF, Rubin LA, et al. Functional variants of OCTN cation transporter genes are associated with Crohn disease. Nat Genet 2004;36:471-5

4 Stoll M, Corneliussen B, Costello CM, et al. Genetic variation in DLG5 is associated with inflammatory bowel disease. Nat Genet 2004;36:476-80.

5 Ma Y, Ohmen JD, Li Z, et al. A genome-wide search identifies potential new susceptibility loci for Crohn's disease. Inflamm Bowel Dis 1999;5:271-8.

6 Rioux JD, Daly MJ, Silverberg MS, et al. Genetic variation in the 5q31 cytokine gene cluster confers susceptibility to Crohn disease. Nat Genet 2001;29:223-8.

7 Wehkamp J, Harder J, Weichenthal M, et al. NOD2 (CARD15) mutations in Crohn's disease are associated with diminished mucosal alphadefensin expression. Gut 2004;53:1658-64.

8 Newman B, Gu X, Wintle R, et al. A risk haplotype in the Solute Carrier Family 22A4/ 22A5 gene cluster influences phenotypic expression of Crohn's disease. Gastroenterology 2005; 128:260-9.

9 Armuzzi A, Ahmad T, Ling KL, et al. Genotypephenotype analysis of the Crohn's disease susceptibility haplotype on chromosome $5 q 31$. susceptibility haplotype

10 Daly MJ, Pearce AV, Farwell L, et al. Association of DLG5 R30Q variant with inflammatory bowel disease. Eur J Hum Genet 2005.

11 Török HP, Glas J, Tonenchi L, et al. Polymorphisms in the DLG5 and OCTN cation transporter genes in Crohn's disease. Gut Published Online First: 14 June 2005. doi:10.1136/gut.2005.066340.

12 Noble C, Nimmo ER, Drummond H, et al. DLG5 variants do not influence susceptibility to inflammatory bowel disease in the Scottish population. Gut Published Online First: 20 April 2005. doi:10.1136/gut.2005.066621.

\section{EDITOR'S QUIZ: GI SNAPSHOT}

\section{An unusual cause of rectal bleeding in a patient with rheumatoid arthritis}

\section{Clinical presentation}

A 75 year old woman presented with rectal bleeding of 10 days' duration. She had a 26 year history of rheumatoid arthritis and duodenal ulcer. Medications included prednisolone $5 \mathrm{mg}$, famotidine $20 \mathrm{mg}$, and indomethacin suppositories $100 \mathrm{mg}$, all daily. On examination, there was no tenderness in the abdomen. Haemoglobin was $10.0 \mathrm{~g} / \mathrm{dl}$. Colonoscopy revealed a diaphragm-like stricture with circumferential ulcer in the rectum (fig 1). Biopsies showed mild non-specific inflammation without granulomas and vasculitis. Culture of stool and biopsies were negative. Tuberculin test was negative.

\section{Question}

What is the diagnosis?

See page 1071 for answer

This case is submitted by:

A Hokama, K Tanaka, M Nakamoto, N Uchima, F Kinjo, A Saito First Department of Internal Medicine, University of the Ryukyus, Okinawa, Japan

Correspondence to: $\operatorname{Dr}$ A Hokama, First Department of Internal Medicine, University of the Ryukyus, 207 Uehara, Nishihara, Okinawa 903-0215, Japan; hokama-a@med.u-ryukyu.ac.jp

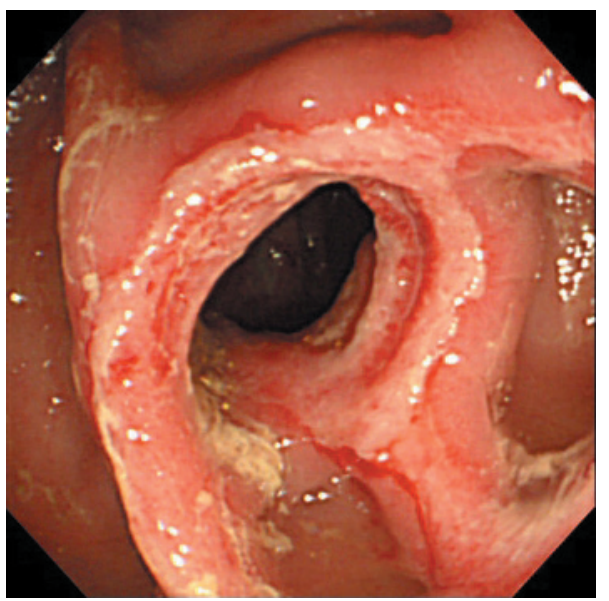

Figure 1 Colonoscopy showing a diaphragm-like stricture with circumferential ulcer in the rectum. 